\title{
Confirmation of association between multiple sclerosis and CYP27B1
}

\author{
Emilie Sundqvist ${ }^{\star, 1}$, Maria Bäärnhielm ${ }^{2}$, Lars Alfredsson ${ }^{2}$, Jan Hillert ${ }^{3}$, Tomas Olsson ${ }^{1}$ and Ingrid Kockum ${ }^{1}$
}

Multiple sclerosis, MS (OMIM No. 126200), is a complex inflammatory disease that is characterized by lesions in the central nervous system. Both genes and other environmental factors influence disease susceptibility. One of the environmental factors that has been implicated in MS and autoimmune disease, such as type 1 diabetes, is vitamin D deficiency, in which patients have lower levels of 25-hydroxyvitamin $\mathrm{D} 3\left(25-\mathrm{OHD}_{3}\right)$ in blood than do controls. Previtamin $\mathrm{D}_{3}$ is produced in the skin, and turned into $25-\mathrm{OHD}_{3}$ in the liver. In the kidney, skin and immune cells, $25-\mathrm{OHD}_{3}$ is turned into bioactive $1,25(\mathrm{OH})_{2} \mathrm{D}_{3}$ by the enzyme coded by CYP27B1 (cytochrome P450 family 27 subfamily B peptide 1 ) on chromosome $12 q 13.1-3$. 1,25(OH) $)_{3}$ binds to the vitamin $D$ receptor, expressed in T cells and antigen-presenting cells. $1,25(\mathrm{OH})_{2} \mathrm{D}_{3}$ has a suppressive role in the adaptive immune system, decreasing T-cell and dendritic cell maturation, proliferation and differentiation, shifting the balance between T-helper 1 (Th1) and Th2 cells in favor of Th2 cells and increasing the suppressive function of regulatory T cells. Rs703842 in the 12q13-14 region was associated with MS in a recent study by the Australian and New Zealand Multiple Sclerosis Genetics Consortium (ANZgene). We show associations with three SNPs in this region in our Swedish materials (2158 cases, 1759 controls) rs4646536, rs 10877012 and rs 10877015 ( $P=0.01,0.01$ and $3.5 \times 10^{-3}$, respectively). We imputed rs703842 SNP and performed a joint analysis with the ANZgene results, reaching a significant association for rs703842 $\left(P=5.1 \times 10^{-11}\right.$; odds ratio 0.83 ; $95 \%$ confidence interval $\left.0.79-0.88\right)$. Owing to its close association with 25- $\mathrm{OHD}_{3}$, our results lend further support to the role of vitamin D in MS pathology.

European Journal of Human Genetics (2010) 18, 1349-1352; doi:10.1038/ejhg.2010.113; published online 21 July 2010

Keywords: multiple sclerosis; CYP27B1; association study; vitamin D; case-control study; meta-analysis

\section{INTRODUCTION}

Multiple sclerosis, MS (OMIM No. 126200), is an inflammatory disease that is characterized by demyelinating lesions in the brain or spinal cord, as well as by axonal loss and progressive buildup of sclerotic plaques. It has a complex disease etiology, in which both genetic and environmental factors influence disease susceptibility. To date, the strongest genetic factor found is the HLA-DRB1 ${ }^{\star} 15$ haplotype (DQB $\left.1^{\star} 0602, \mathrm{DQA} 1^{\star} 0102, \mathrm{DRB} 1^{\star} 1501, \mathrm{DRB} 5^{\star} 0101\right)$. Many other genes with more modest effects on disease risk have been found through candidate gene approaches and genome-wide association studies.

There is an apparent geographical pattern in MS prevalence, with prevalence increasing with increasing latitude. This has been observed in the United States, Europe and Australia. ${ }^{1}$ The level of solar radiation and UV radiation has been implicated as the cause of this difference in prevalence. ${ }^{2,3}$ There is also a month-of-birth effect in MS susceptibility, with fewer cases born during autumn months and more cases born in spring compared with healthy controls, ${ }^{4,5}$ which could be linked to differences in climate during the year, including sun exposure.

Recently, the Australian and New Zealand Multiple Sclerosis Genetics Consortium, ANZgene, published their findings from a genome-wide association study on a total of around 2900 cases and 5700 controls. Besides confirming the association of a number of previously known genes, they also found associated SNPs on chromosomes $12 q 13-14$ and $20 q 13$. The $12 q 13-14$ region contains 17 known genes, including CYP27B1 (cytochrome P450 family 27 subfamily B peptide 1). In the combined cohort, rs703842, which lies $1.76 \mathrm{~kb}$ upstream of CYP27B1, was associated with MS $\left(P=5.4 \times 10^{-11}\right){ }^{6}$

Association with $C Y P 27 B 1$ has been seen in autoimmune diseases; rs4646536 $(+2838 \mathrm{~T}>\mathrm{C})$, which is in high linkage disequilibrium (LD) with rs703842, and $\mathrm{rs} 10877012(-1260 \mathrm{C}>\mathrm{A})$ have previously been associated with type 1 diabetes, ${ }^{7,8}$ Hashimoto's thyroiditis and Graves' disease. $^{9}$ Rs4646536 and rs703842 have been associated with 25-hydroxyvitamin D3 $\left(25(\mathrm{OH}) \mathrm{D}_{3}\right)$ levels in Canadian twins, in which at least one of the twins suffered from MS. ${ }^{10}$

Vitamin D3 is naturally produced in the skin after UV B radiation, and can also be supplied in the diet. The enzyme 25-hydroxy-vitamin D-1 alpha hydroxylase, coded by the gene CYP27B1, hydroxylates the inactive form of vitamin $\mathrm{D} 3,25-\mathrm{OHD}_{3}$, into its bioactive form, 1,25-dihydroxyvitamin $\mathrm{D} 3\left(1,25(\mathrm{OH})_{2} \mathrm{D}_{3}\right)$. This means that the final level of active vitamin D3 is dependent on both environmental factors, such as sun exposure and diet, and genetic factors such as the CYP27B1 gene. Bioactive vitamin D3 can bind to the vitamin D receptor (VDR) and has a regulatory role in calcium metabolism, as well as in immune functions.

Stimulation with vitamin D3 leads to decreased expression of MHC class II molecules and co-stimulators in B cells, as well as to decreased

\footnotetext{
${ }^{1}$ Neuroimmunology Unit, Department of Clinical Neuroscience, Center for Molecular Medicine, Karolinska Institutet, Stockholm, Sweden; ${ }^{2}$ Institute of Environmental Medicine, Karolinska Institutet, Stockholm, Sweden; ${ }^{3}$ Department of Clinical Neuroscience, Center for Molecular Medicine, Karolinska Institutet, Stockholm, Sweden

${ }^{*}$ Correspondence: E Sundqvist, Neuroimmunology Unit, Department of Clinical Neuroscience, Center for Molecular Medicine L8:04, Karolinska Institutet, Stockholm 17176, Sweden. Tel: +46 85177 6258; Fax: +46 85177 6248; E-mail: Emilie.Sundqvist@ki.se

Received 4 December 2009; revised 19 March 2010; accepted 4 June 2010; published online 21 July 2010
} 
proliferation, differentiation and cytokine production and immune response in T cells. ${ }^{11,12}$ A theory is that the production of CYP27B1, as well as activation of vitamin D3 by $\mathrm{T}$ cells and dendritic cells in extralymphoid tissues, might increase the T-cell programing of antigen-specific response, the so-called T-cell homing. ${ }^{13}$ Overall, the findings support a suppressive role of vitamin D3 in the adaptive immune system.

Low levels of vitamin D in serum are associated with a higher risk of developing MS, ${ }^{14}$ and several studies show that MS patients have lower levels of $25-\mathrm{OHD}_{3}$ compared with controls. ${ }^{11}$ A recent study showed that high circulating $25-\mathrm{OHD}_{3}$ levels among MS patients were associated with improved regulatory T-cell function. It also had an impact on T-helper (Th) cells, shifting the balance in favor of Th2 cells. $^{15}$

On the basis of collective data from previous studies on vitamin D, sun exposure and inflammatory diseases, we decided to investigate whether variants in the CYP27B1 gene were associated with MS in a Swedish population.

\section{MATERIALS AND METHODS}

\section{Data set}

Swedish cohort 1. Swedish cohort 1 consisted of 1013 subjects (727 female) and 1215 blood donor controls (768 female). Patients were recruited from Danderyds Hospital and from Karolinska University Hospital in Huddinge and Solna, in the Stockholm County. Controls were blood donors who visited any of three blood donation facilities in the Stockholm area in 2001 and 2004/2005. Blood was consecutively sampled and resampling was avoided by collecting within 3 months (during which time blood donors were advised not to donate a second time) in 2001 or by explicitly asking whether the sample had been obtained previously (in 2004 and 2005). Controls were not screened for MS. Information about the study, along with questions regarding previous participation and ethnic origin of parents, was presented to donors both orally and in written form. ${ }^{16}$

Swedish cohort 2. These cases and controls are part of an ongoing populationbased case-control study called EIMS (Epidemiological Investigations in Multiple Sclerosis), which has been described previously. ${ }^{17}$ In total, 664 patients (472 females, one unknown sex) and 544 controls ( 406 females) were available for genotyping. Patients and controls are recruited from all over Sweden. Controls are matched for age, sex and residential location.

Swedish cohort 3. Patients $(n=481,347$ female) were recruited from Karolinska University Hospital (Solna) and from clinics all over Sweden.

In total, 2158 cases (1546 females) and 1759 controls (1174 females) were included and available for genotyping. All cases fulfilled the MacDonald criteria. Possible overlaps between cohorts were excluded using the national personal identification number for cases. All participants were of Scandinavian ancestry. Oral or written informed consent was obtained from all participants. The Ethical Committees at Karolinska University Hospital, Huddinge, and Karolinska University Hospital, Solna, approved the study.

\section{DNA extraction}

DNA was extracted from whole blood using standard procedures. DNA concentrations were measured using Picogreen TM fluorescence or with an ND-1000 Spectrophotometer (NanoDrop Technologies Inc., Wilmington, DE, USA).

\section{SNP selection}

SNP markers were selected for genotyping by running the tagging algorithm ${ }^{18}$ implemented in Haploview 3.32 based on data from the HapMap project using $r^{2}=0.9$ and LOD 3.0. (http://www.hapmap.org/, release 21/phaseII, NCBI B35 assembly, dbSNP b125). Two additional SNPs were included either because of reported association with type 1 diabetes (rs10877012) or because of a proposed involvement in MS susceptibility (rs4646536) ${ }^{8,10}$ A complete list of included SNPs is shown in Table 1.

\section{Genotyping}

Two methods were used for allelic discrimination in the Swedish cohorts (see Table 1). Rs10877012 and rs4646536 were genotyped using Taqman SNP assays from Applied Biosystems (Foster City, CA, USA), as described previously. ${ }^{19}$ Six SNPs, including rs4646536, were genotyped in all cohorts using matrix-assisted laser desorption/ionization time-of-flight (MALDI-TOF) mass spectrometry (Sequenom Inc., San Diego, CA, USA), as described previously. ${ }^{16}$ Primer sequences are available on request. One of the tagSNPs (rs11172327), which only tagged itself, was excluded from the analysis because of low success rate $<75 \%$; however, this marker was also monomorphic in our samples.

\section{Imputation and statistical analysis}

Association of genotypes in the Swedish samples was tested using the model command in PLINK v1.06 (http://pngu.mgh.harvard.edu/ purcell/plink/ index.shtml). Imputation was performed using Mach 1.0, ${ }^{20}$ accessed through the HapMap Genome Browser (Phase 3 - genotypes, frequencies and LD). The CEU population was used as reference population; the region was set to chromosome 12, $56430000-56470000 \mathrm{~kb}$. Rs8176345 was excluded from the imputation because it was not in Hardy-Weinberg equilibrium among controls. All other settings were kept at default. A fixed-effects (Mantel-Haenszel) meta-analysis was performed using the rmeta package and the meta.MH command in R 2.8.0. A joint $P$-value was obtained from Unphased, using the study population as a covariate. ${ }^{21}$

\section{RESULTS}

Seven SNPs were genotyped in the Swedish cohorts, described in more detail in Table 1 (also see Supplementary Table 1 for detailed

Table 1 Summary of the SNPs genotyped and imputed in the Swedish material

\begin{tabular}{|c|c|c|c|c|c|c|c|c|c|c|c|c|c|c|c|}
\hline \multirow[b]{2}{*}{ SNP } & \multirow[b]{2}{*}{ Alt. name } & \multicolumn{4}{|c|}{ Alleles } & \multicolumn{3}{|c|}{ Cases } & \multicolumn{3}{|c|}{ Controls } & \multirow{2}{*}{$\begin{array}{c}\text { HWE (P-value) } \\
\text { Controls }\end{array}$} & \multicolumn{3}{|c|}{ Association, allelic test } \\
\hline & & Position $^{\mathrm{a}}$ & Minor & Major & Method & Total N & $M A F$ & No. & Total N & $M A F$ & No. & & P-value & $O R$ & $95 \% \mathrm{Cl}$ \\
\hline rs1048691 & & 56439215 & $\mathrm{~T}$ & C & MT-MS & 1938 & 0.222 & 760 & 1722 & 0.226 & 699 & 0.21 & NS & & \\
\hline rs4646537 & & 56443548 & G & $\mathrm{T}$ & MT-MS & 2072 & 0.038 & 161 & 1723 & 0.041 & 141 & 0.36 & NS & & \\
\hline rs4646536 & $+2838 \mathrm{~T}>\mathrm{C}$ & 56444255 & G & $A$ & Taqman/MT-MS & 2064 & 0.324 & 1118 & 1733 & 0.352 & 1008 & 0.79 & 0.01 & 0.88 & $0.80-0.97$ \\
\hline rs8176345 & & 56444825 & $\mathrm{~T}$ & C & MT-MS & 2102 & 0.029 & 121 & 1751 & 0.030 & 101 & 0.02 & NS & & \\
\hline rs2307096 & & 56445011 & & $\mathrm{C}$ & MT-MS & 2040 & 0.00 & & 1708 & 0.00 & & 1.00 & NA & & \\
\hline rs10877012 & $-1260 \mathrm{C}>\mathrm{A}$ & 56448352 & $\mathrm{~T}$ & G & Taqman & 1973 & 0.326 & 1075 & 1682 & 0.354 & 982 & 0.92 & 0.01 & 0.88 & $0.80-0.97$ \\
\hline rs10877015 & & 56454055 & $G$ & $A$ & MT-MS & 2086 & 0.321 & 1129 & 1741 & 0.353 & 1016 & 0.68 & $3.5 \times 10^{-3}$ & 0.87 & $0.79-0.95$ \\
\hline rs703842 & & 56449006 & $G$ & $A$ & Imputed & 2158 & 0.330 & 1209 & 1759 & 0.355 & 1037 & 0.32 & 0.02 & 0.89 & $0.81-0.98$ \\
\hline
\end{tabular}

Abbreviations: MT-MS, MALDI-TOF MS; NS, nonsignificant.

Total number of cases and controls genotyped, the minor allele frequency for each group and the number of individuals carrying the minor allele. aNCBI build 36.3 . 


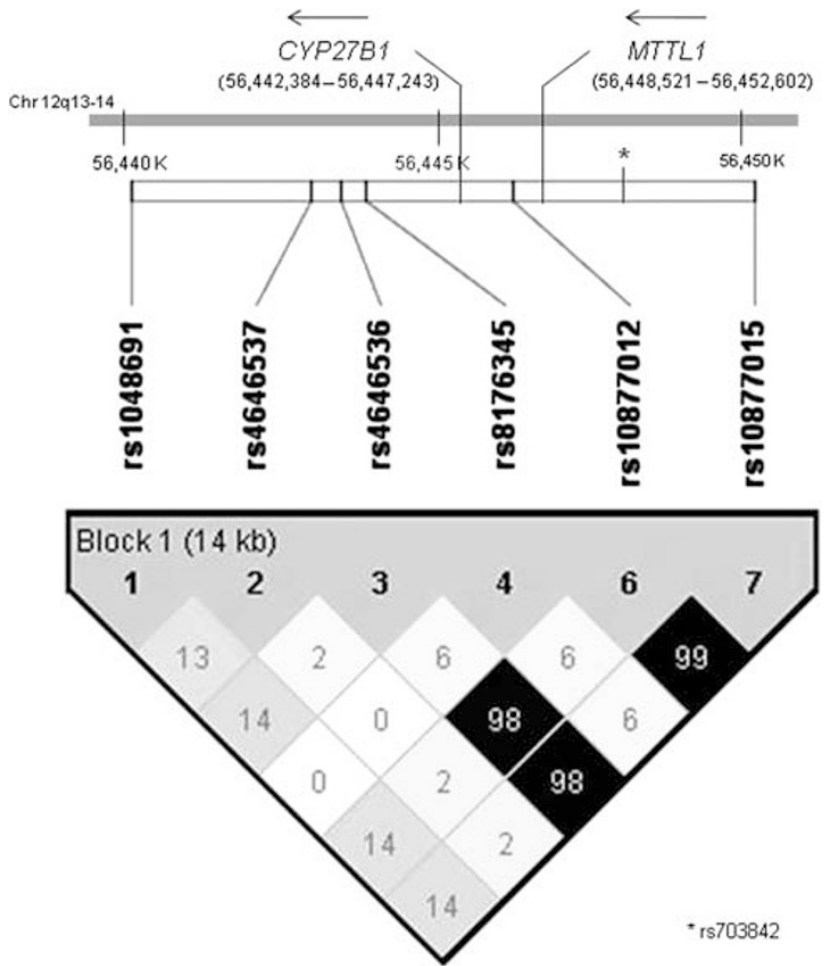

Figure 1 Linkage disequilibrium (LD) plot. A plot showing the LD structure of the six markers genotyped in the Swedish cohorts in the 12q13-14 region. The location of the imputed SNP, rs703842, is marked with an asterisk. The LD in the region of the tested genes is given as $r^{2}$, and block structure was analyzed using the four-gamete rule.

information on each of the three cohorts). All SNPs, except rs8176345, were in Hardy-Weinberg equilibrium among unaffected controls. Three SNPs genotyped in the Swedish cohorts were associated with MS, rs4646536, rs10877012 and rs10877015, with $P$-values of 0.01 , 0.01 and $3.5 \times 10^{-3}$, respectively. They all had a reduced minor allele frequency among cases, with odds ratios (OR) of $0.88,0.88$ and 0.87 , respectively, for the minor allele. When we studied the $\mathrm{LD}$ in the region (Figure 1), and defined the blocks either by the four-gamete rule or solid spine of $\mathrm{LD}$, there were two associated haplotypes, a CTACGA haplotype $\left(P=8.5 \times 10^{-3}\right)$ and a CTGCTG haplotype $\left(P=7.8 \times 10^{-3}\right)$. Defining the blocks by the confidence interval $(\mathrm{CI})$ method yielded similar results.

Imputed SNP and combined analysis

The most associated SNP from the ANZgene study located in the 12q13-14 region was rs703842, at position 55449006, between rs10877012 and rs10877015, run in our own samples. Genotypes from 1759 controls and 2158 Swedish cases were imputed. Other SNPs found in the ANZgene paper could not be imputed in our material. Rs703842 was found to be significantly associated (Table 1) with a $P$-value of 0.02 and an OR of 0.89 (95\% CI 0.81-0.98) for the minor allele, showing the same protective effect found in the ANZgene data sets.

For the combined analysis, genotypes from a total of 6032 cases and 7482 controls were available after imputation (see Table 2). The results from the meta-analysis can be seen in Figure 2. The combined OR for rs703842 was 0.83 (95\% CI 0.79-0.88). The overall association was significant, $P=5.1 \times 10^{-11}$.
Table 2 The different study populations

\begin{tabular}{lccc}
\hline Study & Cases & Controls & Genotyped/imputed \\
\hline ANZgene GWAS & 1618 & 3413 & Genotyped \\
ANZgene replication & 2256 & 2310 & Genotyped \\
Sweden & 2158 & 1759 & Imputed \\
Total $N$ & 6032 & 7482 & \\
\hline
\end{tabular}

The three different study populations used in the combined analysis for rs703842. Partially adapted from ANZgene, 2009.

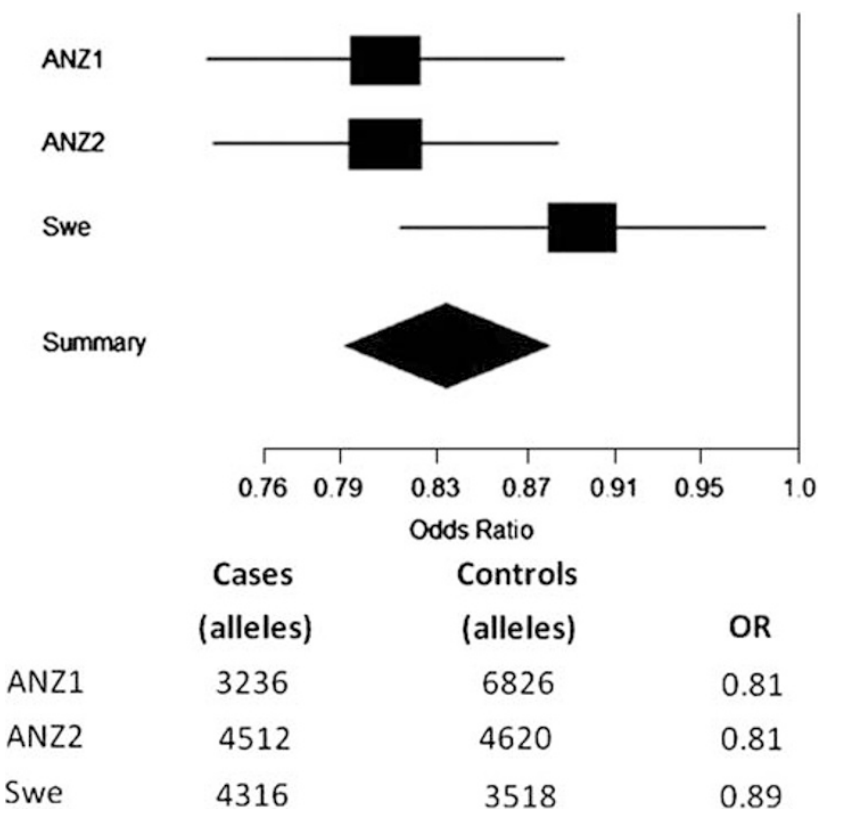

Figure 2 Meta-analysis of the two ANZgene cohorts combined with the Swedish cohorts, with the allele count for cases and controls, and the odds ratio for each group. The combined odds ratio is $0.83(95 \% \mathrm{Cl}$ 0.79-0.88).

\section{DISCUSSION}

In this study, we tested the association of the CYP27B1 gene with MS in a Swedish population. We replicate the previously identified association in the Australian and New-Zealand population (ANZgene 2009). In a joint analysis, the association of rs703842 with MS shows an OR of 0.83 (95\% CI $0.79-0.88)$, reaching a significance of $5.1 \times 10^{-11}$, lending further evidence to the involvement of CYP27B1 and vitamin D3 in MS.

The candidate region on chromosome 12q13-14 studied by the ANZgene consortium contains 17 genes. CYP27B1 is the most likely MS susceptibility candidate, given its importance in vitamin D3 activation and the important role of vitamin D3 in immunological functions. In this study, we have confirmed the association between MS and the CYP27B1 gene. We cannot, however, exclude the possibility that our association with CYP27B1 is caused by LD with some other marker in the 12q13-14 region, such as polymorphisms in the MTTL1 gene. This study was a case-control study; although it has been performed in the fairly homogeneous Swedish population, it is possible, although unlikely, that our observed association is due to population stratification between cases and controls. This could be corrected for by performing an association analysis in a family-based 
material or by using genomic controls, which unfortunately were not available for the included individuals. ${ }^{22}$

Several studies show that MS patients have lower levels of $25-\mathrm{OHD}_{3}$ compared with controls. ${ }^{11}$ In an Australian study, low levels of $25-\mathrm{OHD}_{3}$ could be linked to increased disability, likely caused by decreased sun exposure due to disability. ${ }^{23}$ It has also been shown that there is an association between SNPs in the CYP27B1 region and $25(\mathrm{OH}) \mathrm{D}_{3}$ levels. ${ }^{10}$

Lower levels of $25-\mathrm{OHD}_{3}$ and $1,25(\mathrm{OH})_{2} \mathrm{D}_{3}$ have also been seen in type 1 diabetes patients, and they also had a lower expression of CYP27B1 mRNA compared with controls. This reduction in expression could be linked to the GG variant of the rs10877012 polymorphism. ${ }^{24}$ In our own material, there was a significant difference in the number of GG homozygotes between cases and controls (cases frequency: 0.46; controls frequency: $0.42, P=0.02$ ); therefore, we would expect a similar result in terms of CYP27B1 expression in our own material.

One of the important immune genes regulated by vitamin $\mathrm{D}$ is the HLA class II locus, in which there is a VDR response element in the HLA-DRB1 promoter region that is highly conserved in the HLA$\mathrm{DRB}^{\star} 1501$ haplotype, which is the most important risk gene in MS. It was also shown that vitamin D3 had an effect on HLA-DRB1 ${ }^{\star} 1501$ expression in vitro. However, the exact mechanism for the interaction between vitamin D3 and HLA and the determination of the tissues or cells in which it takes place remain unknown. ${ }^{25}$

Lower exposure to sunlight and low expression of CYP27B1 mRNA will likely influence the amount of available active vitamin D3 in the body. In individuals with other genetic risk factor(s) and possibly exposed to other environmental factor(s), this could, through the effects of vitamin D3 on the immune system, lead to the development of MS or other autoimmune disease.

The results from studies on type 1 diabetes and CYP27B1 genotype and expression levels show that CYP27B1 expression could have a role in autoimmune disease. This should be replicated in MS patients, as well as in studies on the relationship between genotype and $25-\mathrm{OHD}_{3}$ levels. It will also be of value to study possible genetic interactions between CYP27B1, VDR and the HLA complex, in particular HLA$\mathrm{DRB}^{\star}{ }^{\star} 1501$.

\section{CONFLICT OF INTEREST}

Ms Sundqvist reports no disclosures. Dr Bäärnhielm has received unrestricted research support from Biogen Idec and from Sanofi Aventis. Prof. Alfredsson receives research support from the Swedish Medical Research Council (K2007-69X-14973-04-3) and Swedish Council for Working life and Social Research (Dnr 2006-0655). Prof. Hillert has received unrestricted research support from BiogenIdec, MerckSerono and Bayer Schering. Prof. Olsson has received unrestricted research support from BiogenIdec, MerckSerono, SanofiAventis and Bayer Schering. Dr Kockum reports no disclosures.

\section{ACKNOWLEDGEMENTS}

This study was supported financially by Neuropromise (LSHM-CT-2005018637), Swedish Research Council, Swedish Foundation for the neurologically
Disabled, the Söderberg foundation, Bibbi and Niels Jensens foundation, Swedish Council for Working life and Social Research, the Swedish Associations for Persons with Neurological Disabilities and the Montel Williams foundation.

1 Kurtzke JF: Epidemiology of multiple sclerosis. Does this really point toward an etiology? Lectio Doctoralis. Neurol Sci 2000; 21: 383-403.

2 Acheson ED, Bachrach CA, Wright FM: Some comments on the relationship of the distribution of multiple sclerosis to latitude, solar radiation, and other variables. Acta Psychiatr Scand 1960; 35 (Suppl): 132-147.

3 van der Mei IA, Ponsonby AL, Blizzard L, Dwyer T: Regional variation in multiple sclerosis prevalence in Australia and its association with ambient ultraviolet radiation. Neuroepidemiology 2001; 20: 168-174.

4 Willer CJ, Dyment DA, Sadovnick AD, Rothwell PM, Murray TJ, Ebers GC: Canadian Collaborative Study Group. Timing of birth and risk of multiple sclerosis: population based study. BMJ 2005; 330: 120.

5 Bayes HK, Weir CJ, O'Leary C: Timing of birth and risk of multiple sclerosis in the Scottish population. Eur Neurol 2010; 63: 36-40.

6 Australian and New Zealand Multiple Sclerosis Genetics Consortium: Genome-wide association study identifies new multiple sclerosis loci on chromosomes 12 and 20. Nat Genet 2009; 41: 824-830.

7 Bailey R, Cooper JD, Zeitels L et al: Association of the vitamin D metabolism gene CYP27B1 with type 1 diabetes. Diabetes 2007; 56: 2616-2621.

8 Lopez ER, Regulla K, Pani MA, Krause M, Usadel KH, Badenhoop K: CYP27B1 polymorphisms variants are associated with type 1 diabetes mellitus in Germans. J Steroid Biochem Mol Biol 2004; 89-90: 115-157.

9 Lopez ER, Zwermann O, Segni M et al: A promoter polymorphism of the CYP27B1 gene is associated with Addison's disease, Hashimoto's thyroiditis, Graves' disease and type 1 diabetes mellitus in Germans. (B) Eur J Endocrinol 2004; 151: 193-197.

10 Orton SM, Morris AP, Herrera BM et al: Evidence for genetic regulation of vitamin D status in twins with multiple sclerosis. Am J Clin Nutr 2008; 88: 441-447.

11 Smolders J, Damoiseaux J, Manheere P, Hupperts R: Vitamin D as an immune modulator in multiple sclerosis, a review. J Neuroimmunol 2008; 194: 7-17.

12 Gorman S, Kuritzky LA, Judge MA et al: Topically applied 1,25-dihydroxyvitamin D3 enhances the suppressive activity of CD4+CD25+ cells in the draining lymph nodes. J Immunol 2007; 179: 6273-6283.

13 van Etten E, Stoffels K, Gysemans C, Mathieu C, Overbergh L: Regulation of vitamin D homeostasis: implications for the immune system. Nutr Rev 2008; 66 (Suppl 2): S125-S134.

14 Munger KL, Levin LI, Hollis BW, Howard NS, Ascherio A: Serum 25-hydroxyvitamin D levels and risk of multiple sclerosis. JAMA 2006; 296: 2832-2838.

15 Smolders J, Thewissen M, Peelen E et al: Vitamin D status is positively correlated with regulatory T cell function in patients with multiple sclerosis. PLoS One 2009; 4: e6635.

16 Roos IM, Kockum I, Hillert J: The interleukin 23 receptor gene in multiple sclerosis: a case-control study. J Neuroimmunol 2008; 194: 173-180.

17 Hedström AK, Bäärnhielm M, Olsson T, Alfredsson L: Tobacco smoking, but not Swedish snuff use, increases the risk of multiple sclerosis. Neurology 2009; 73: 696-701.

18 de Bakker PI, Yelensky R, Péer I, Gabriel SB, Daly MJ, Altshuler D: Efficiency and power in genetic association studies. Nat Genet 2005; 37: 1217-1223.

19 Ekelund $\mathrm{E}$, Saaf $\mathrm{A}$, Tengvall-Linder $\mathrm{M}$ et al: Elevated expression and genetic association links the SOCS3 gene to atopic dermatitis. Am J Hum Genet 2006; 78: 1060-1065.

$20 \mathrm{Li} \mathrm{Y,} \mathrm{Abecasis} \mathrm{GR:} \mathrm{Mach} \mathrm{1.0:} \mathrm{rapid} \mathrm{haplotyoe} \mathrm{reconstruction} \mathrm{and} \mathrm{missing} \mathrm{genotype}$ inference. Am J Hum Genet 2006; S79: 416.

21 Dudbridge F: Likelihood-based association analysis for nuclear families and unrelated subjects with missing genotype data. Hum Hered 2008; 66: 87-98.

22 Lange EM, Sun J, Lange LA et al: Family-based samples can play an important role in genetic association studies. Cancer Epidemiol Biomarkers Prev 2008; 17: 2208-2214.

23 van der Mei IA, Ponsonby AL, Dwyer T et al: Vitamin D levels in people with multiple sclerosis and community controls in Tasmania, Australia. J Neurol 2007; 254: 581-590.

24 Ramos-Lopez E, Brück P, Jansen T, Pfeilschifter JM, Radeke HH, Badenhoop K: CYP2R1-, CYP27B1- and CYP24-mRNA expression in German type 1 diabetes patients. J Steroid Biochem Mol Biol 2007; 103: 807-810.

25 Ramagopalan SV, Maugeri NJ, Handunnetthi L et al: Expression of the multiple sclerosis-associated MHC class II allele HLA-DRB1*1501 is regulated by vitamin D. PLoS Genet 2009; 5: e1000369.

Supplementary Information accompanies the paper on European Journal of Human Genetics website (http://www.nature.com/ejhg) 